\title{
Optimized Dynamic Routing in Multimedia Vehicular Networks
}

\author{
Dr. Joy Iong Zong Chen \\ Professor, \\ Department of Electrical Engineering, \\ Da-Yeh University, \\ Taiwan. \\ Email id: jchen@mail.dyu.edu.tw
}

\author{
Dr. S. Smys \\ Professor, \\ Department of CSE, \\ RVS Technical Campus, \\ Coimbatore, India. \\ Email id: smys375@gmail.com
}

\begin{abstract}
Intelligent transport system is one of thriving research domain and in particular multimedia vehicular networks gains more attention. The deployment of multimedia services in vehicle ad-hoc networks (VANETs) provides real time support to users and provides better traffic management with high safety measures. Most of the vehicles has multimedia applications such as weather sensors, real time map applications etc., and it requires adequate resources to process. Resource allocation to a static network is simple and various routing models are evolved. In case of dynamic network like VANETs, routing is still in progress in order to obtain a better model. Routing directly related to quality of services of network and user, it is essential to develop a better dynamic routing strategy for multimedia wireless networks. The proposed work aims to provide an optimized dynamic routing strategy for multimedia networks. For efficient routing, k-means clustering is used to organize the clusters and exchanges the routing information and inverted ant colony optimization is used to obtain the optimal path for multimedia access. Proposed model is experimentally verified and compared to conventional ant colony optimization and grey wolf optimization in terms of parameters such as end to end delay, throughput, target used, average computation time and efficiency.
\end{abstract}

Keywords: - Multimedia Vehicular Networks (MVANets), K-means clustering, Inverted Ant Colony optimization (IACO)

\section{Introduction}

Vehicular Networks are used for monitoring and data sharing of traffic on the urban areas. Vehicles use their connections for improving their safety and also provide some internet applications such as online gaming, video streaming, and social networks. Moreover, the cars are traveling from one access point (AP) to another access point from a network point of view. This brings the continuous variations in APs, as the vehicle travels along the road. The main features of the vehicular network are providing coverage throughout the city because the vehicles move around the whole city. It captures the details of several cities and it monitors the diverse situations like car accidents, traffic jams, animals crossing on the road, and potholes. the vehicular network consists of vehicle nodes that are different from other nodes. The main aim of the vehicular network is to disseminate messages picked up from a local gateway sensor. Figure 1 shows the diagrammatic representation of the vehicular network. In vehicular networks, providing high-quality multimedia services is a challenging task and high-cost is a notable limitation. With the increase of wireless devices and wireless network technologies, it provides a big challenge for delivering multimedia services like online games, street viewing, and classic or 3D video. Multimedia streaming is initiated at each local server to pre-fetch and buffer the stream data. In Vehicular networks, vehicles exchange their real-time data to avoid accidents. For efficient delivery of multimedia streaming techniques that should be incorporated into the entertainment systems and vehicular networks. That streaming can be either ondemand or live streaming. For live streaming, interactive video conferencing the media should be compressed and transmitted to the media players via servers and networks. 
Vol.02/ No. 03

Pages: $174-182$

https://www.irojournals.com/itdw/

DOI: https://doi.org/10.36548/jitdw.2020.3.005

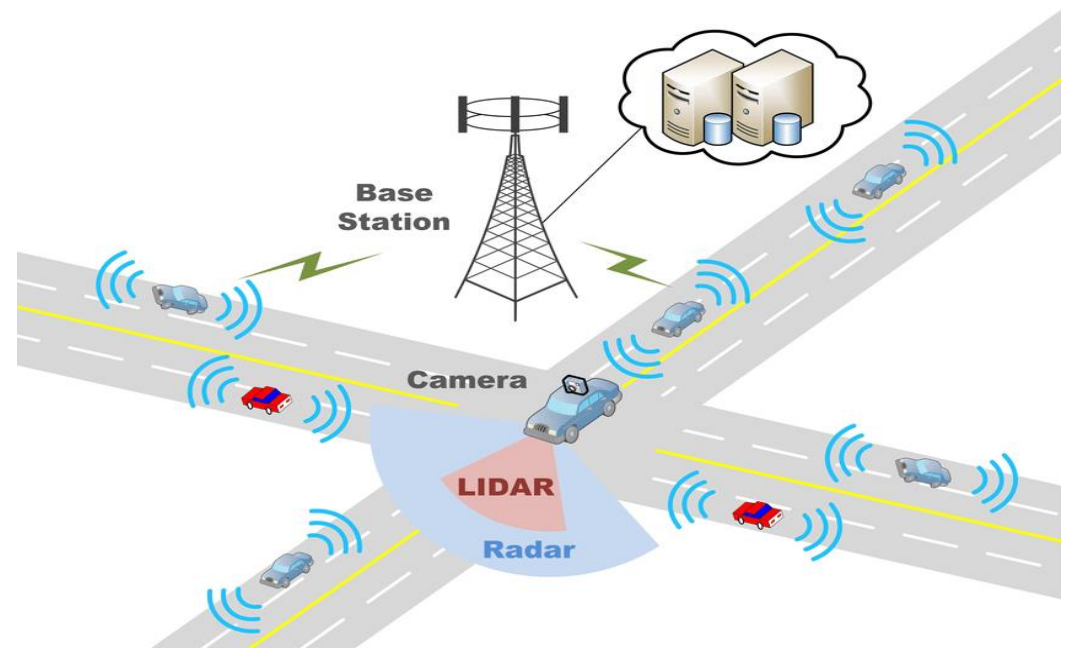

Fig.1 Vehicular Networks

In on-demand streaming, all the media files should be saved in the database for future use. Then, the particular files are transmitted from the database whenever the file services are needed by the clients. This streaming is classified into three types such as HTTP Streaming, True Streaming, and Clientless Streaming. HTTP Streaming involves sending multimedia files to clients through web servers. True Streaming is used for playing multimedia files via independent streaming servers. This type of streaming defines the Real-Time Protocol (RTP) and is specially designed for real-time streaming purposes and it is similar to FTP and HTTP protocol. In Clientless Streaming, the users play the stream files with the help of a Java player. This Java player is not present on the client side initially and it is delivered based on the client request when it is needed.

Figure 2 shows the multimedia services in vehicular networks. The main advantage of the streaming technique is anyone can stream media at anytime, anywhere at any place. To transmit digital media, it is transferred using P2P techniques. While transmitting the digital data, it requires high bandwidth in the vehicular environment. It is better to increase the Quality of Experience (QoE) and decrease the bandwidth for video streaming transmission. Various wireless technology has been developed to support delivering the multimedia content in vehicular networking infrastructure. These techniques are classified into vehicle-to-vehicle communications (V2V), vehicle-to-roadside unit (V2R), vehicle-to-infrastructure (V2I). All the vehicular applications need to support high-speed mobility and able to provide drivers with information and to maintain a connection between the vehicle and the Roadside units (RSU).

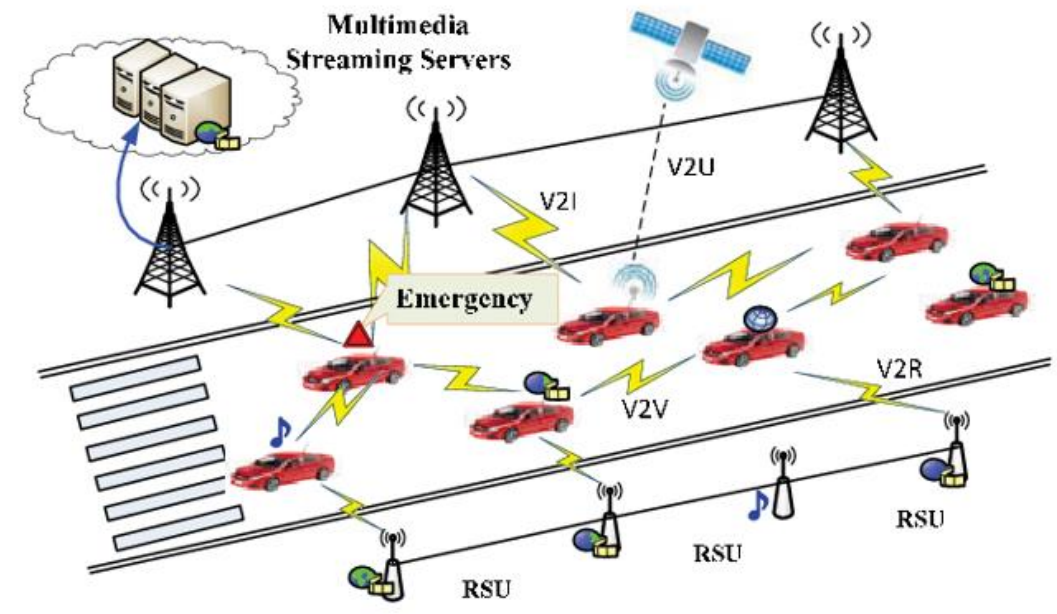

Fig. 2 Multimedia Services in Vehicular Networks 
Journal of Information Technology and Digital World (2020)

Vol.02/ No. 03

Pages: $174-182$

https://www.irojournals.com/itdw/

DOI: https://doi.org/10.36548/jitdw.2020.3.005

The IEEE 802.11p communication protocol provides standard and support to wireless access in the vehicular environment as task groups. Vehicular Networks can equip vehicles with the most advanced and embedded devices. It includes diverse sensors that increase the capabilities of the vehicles and provide the computation capabilities thereby collect the multimedia content in the form of a vehicular network. For the vehicles equipped with wireless access, all the communications are carried out using the Internet. The vehicles that have DSRC allowed for various categories like V2R, V2V, and V2R, etc., But it is also possible with Wi-Fi devices. The vehicles can be equipped with smart sensors and actuators to provide data-acquisition and decisionmaking. The vehicular network has the greatest advantage in the future because the need for communication between the vehicles and the outside world would be a great demand.

\section{Related Works}

An intense research analysis is performed to obtain the issues in existing vehicular network models. The multipath TCP architecture in vehicular networks was suggested by Kannan.et.al. [1] for infotainment multimedia applications. It is presented for semantics quality of service of inbred multimedia to grab the brim-full precedence to achieve resources that are self-governing and also to dispense services of transport based upon the Inspection process of packets. Because of diverse wireless networks, the multipath and multi-homing potentialities of mobile junctions likely to sheer portability in the transport layer. Jiau et.al. [2] proposed a framework which uses a protocol called MPTCP to magnify the aids and DPI is to scrutinize the data of video for spotting the frames. They illustrated two operations to improvise the service of video streaming are operations of MPTCP and rules of DPI. The multi-fold paths are used in Changqiao Xu et.al. [3] MPTCP model to elongate the bandwidth deployment and DPI are elucidated using sub-flows to refashion the traffic to be forwarded. It is obtained as a prim protocol till receipt of mislaid packets, MPTCP shields the unordered packets. The execution of lodged architecture signifies better outcomes in the structure of the vehicular network. Offered extension of the framework is to collude with the criterion of Autonomic Computing.

Huang et.al. [4] research reports about the streaming of multimedia based on hierarchical fog computing for vehicular environments that lessens dormancy also shrinks the use of bandwidth using the Internet. Simulation is undergone for the appraisal of initiated framework and flavour of video cascading. The examined conclusion amplifies the finer quality of experience and service for the proposed system. It set aside the cloud implication to be negligible for obeying the demands of clients actually for coherent bandwidth handling. In terms of level one, the server distributes the requests and the next level heed based on hierarchy. The SF acquires narration from the cloud in the procedure of content analysis presented by Kharel et.al. [5] further proceeded the SF to recognize which are the contents yet to be hoarded in the PF. This PF has localized inmost the vehicle with slender resources such as the ability for functioning and storing. For the demonstration, video data is contemplated as the content of media. During simulation it presumes no barrier allying RSU and LoS and Wimax BS viewed as immobile. The network performance is much better in the non-burdened context of the network. Research work illustrated the network staging in a cram-full circumstance.

Later modelling simulation is reported in Zhou Su et.al. [6] research work which observes the site deeds as concert estimation based on the web scenarios. The major reviews are QoE and QoS also considers some parameters are a variation of packet delay, Detection of packet loss, delay of end to end-user, and a score of the visual mean.The Information-Centric Networking - based vehicle to cloud delivery was initiated by Wei Quan et.al. [7] in urban vehicular networks for multimedia streaming. They apotheosis that squints in the vehicular network can acquire hunks in multimedia through $\mathrm{V} 2 \mathrm{C}$ methodology to raise the quality of dispatch. This research offers a flimsy approach of multimedia inclination to escort the web system to regulate the forwarding way. One and all network junction call to be provisioned by Lai et.al. [8] along with the accelerating engine of ICN supports the process of ICN based forwarding. Actively the ICN samples are generated which can yield streaming services of information-centric multimedia for vehicles.

An essential universally disseminated forwarding engine reported in Yang et.al. [9] research model to ground upon ICN in notable infrastructures encompassing vehicles, servers of multimedia, cloud, and roadside units. The spy of the vehicle could have prevailed from the impending caches and packets are created by some extremity inlay, remitted ahead paths adopted. The node ought to connect with IC overlay logically and the information is the relay to additional nodes by member constantly. As an auxiliary cloud is advanced with assets comptroller. They calculate the average ratio of transmissions by using various paths probabilities and the 
Journal of Information Technology and Digital World (2020)

Vol.02/ No. 03

Pages: $174-182$

https://www.irojournals.com/itdw/

DOI: https://doi.org/10.36548/jitdw.2020.3.005

complete responded system requests. Xia et.al.[10] reported an adaptive multimedia data forwarding scheme in vehicular ad-hoc networks which improves the privacy of user data. Considering the security and privacy issues, the network status and resource allocation strategies are modified using adaptive algorithm. Proposed model attains better performance over multimedia data compared to conventional static models. Similar research work is reported in Poongodi et.al.[11] research model which provides data forwarding using authentication group key model.

The role of machine learning in vehicular ad-hoc networks is unavoidable and various research models are evolved in the past decade. Most of the research models uses artificial neural networks, back propagation neural network for network classification. Recently Noura Aljeri et.al.[12] reported the issues in conventional neural network based vehicle ad-hoc networks using two-tier machine learning model for better handover management. Proposed intelligent vehicular networks systems reduces the limitations of conventional models using recurrent neural network and Markov model by selecting the access points and vehicle flow projection. In order to optimize the performance of machine learning models, optimization schemes are introduced in VANETs. Salim Bitam et.al.[13] proposed a bee life-based routing model for multi constraints environment as multicast optimized routing model. The resultant features after classification is considered in the research work and provides minimal path from user to network which improves the QoS factors.

Muhammad Fahad et.al.[14] proposed a clustering algorithm based on grey wolf optimization for VANET, which selects the cluster heads for transmission and provides continuous service to user. Sadip Midya et.al.[15] proposed a multi-objective optimization technique which uses particle swarm optimization and genetic algorithm for effective resource allocation along with task scheduling. This hybrid approach attains better performance in various aspects such as end to end delay, bandwidth. From the above survey it is observed that effective routing with minimum path could improve the network performance and quality of services. Most of the research works are focused over single algorithm to attain minimum satisfactory level performances. Considering these issues, the proposed model is intended to developed as dynamic routing model for multimedia vehicular networks.

\section{Proposed work}

Dynamic routing by identifying the shortest path is mathematically formulated in this section. In order to obtain the desired performance, k-means clustering is used in the initial stage of the proposed work and inverted ant colony optimization is implemented in the final stage to obtain minimal path. This improves the network efficiency and other factors. k-means is selected due to its effective cluster selection strategy and ant colony is used to obtain the shortest path within short duration time. In VANETs, depend upon the number vehicles which are connected to RSUs justifies the data collection reliability. Since the data collection varies in its volume based on traffic, resource demands and dynamic nature of the network. In order to obtain essential data, identifying the neighbour nodes is an essential process. Since it reduces the network complexity by selecting nearby nodes to transmit the data periodically, improves the network performances. In this process each adjacent neighbours are selected and a list is prepared as neighbour table $n(\varphi)$. The neighbour presence is confirmed by periodic beacon sent to all vehicles present in the communication range. Beacon signal includes the details of sender, location and velocity through navigation systems. Once the beacon is received, the destination vehicle node summarizes the information to be sent in the neighbour table and it is obtained as

$$
I(u)=\left[I_{n}, h^{(x, y)}, u, \tau\right]
$$

where $I$ is the information to be sent, $I_{n}$ is the unique Id of sender, location of the source and destination is represented as $h^{(x, y)}, u$ represents the neighbour and $\tau$ is the threshold timer. The minimum value for timeout is assumed to be five seconds and in that mean time the information table of neighbours are need to be updated based on the beacon messages. Using this process, destination identifies the source from the neighbour table. Once the timer has expired the neighbour entry is removed from the table.

Cluster head selection is an important process and using $\mathrm{k}$ means clustering, based on the factors such as velocity, delay and identifier, the cluster heads are selected. Generally, the identifier is considered as an unsigned integer value which is used to identify the vehicles in the network. Based on the weight metric, the relative velocity is measured. The velocity is assumed as zero for congested regions and varied for normal regions. In order to 
Journal of Information Technology and Digital World (2020)

Vol.02/ No. 03

Pages: $174-182$

https://www.irojournals.com/itdw/

DOI: https://doi.org/10.36548/jitdw.2020.3.005

increase the cluster lifetime and manage the cluster assignments, velocity difference is used as it determines the optimal speed of the vehicles. Delay defines the details of closest vehicles and its neighbour details. Considering all the three factors, it is formulated as

$$
\begin{aligned}
& u=\frac{1}{\rho} \sum_{\omega=n(\varphi)} v(\omega) \\
& w(\varphi)=I(\varphi) \alpha+|v(\varphi)-u| \beta \frac{1}{\rho} \sum_{\omega=n(\varphi)} d(\varphi, \omega)
\end{aligned}
$$

where the degree of $\varphi$ is represented as $\rho$ and its values are $\rho=|n(\varphi)|$, the vehicle identifier is given as $I(\varphi)$ along with its relative velocity of $v(\varphi)$. The relation between the vehicle and neighbours are expressed in terms of beacon delay as $d(\varphi, \omega)$. The values $\alpha$ and $\beta$ are constants which are used as factors of dominant criterion. In order to create clusters, each vehicle is considered as predominant with lowest weight factors. If a vehicle has lowest weights, then it is considered as cluster head. The vehicle weights are managed based on the identifier messages. In some cases, if one or more neighbour cluster heads are identified, then the joining message will be forwarded to both low value cluster heads and creates a cluster at a distance of 3hops.

Once the cluster head is selected, using broadcasting message cluster members are assigned in the network. The message includes details such as source identifier, $\mathrm{CH}$ flag status, usually it is on for member assignment, current location, relative velocity and weight. Similarly, once the message is received, the location and velocity values are updated by the neighbour vehicle in its own neighbour table. If the cluster head value is high, a joining message will be forwarded to the network to change its responsibilities into a cluster member by comparing the weights of the cluster. In case, the identifiers have equal weights, location and velocity values are updated in the neighbour table. The corresponding source id is added to the cluster table. Meanwhile for inter communication failure, periodic check is performed by the vehicles to obtain the movement of neighbour with in the network. If the vehicle moves away from the cluster range, initially a beacon is sent to obtain response. Even if there is no response for beacon after 5 seconds, then the vehicle information is removed from neighbour table.

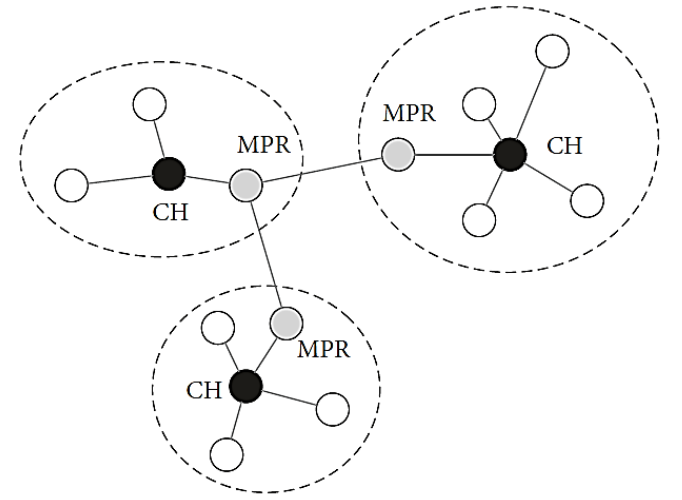

Fig. 3 Cluster formation in Multimedia VANET

If the table values are updated and cluster heads are selected, it is essential to obtain shortest path to transfer information between the vehicles or servers. For this process, inverted ant colony (IACO) is utilized in the proposed model. Based on the pheromone information and heuristic information, the network routing is established in IACO. In order to relate the optimization model and VANET, ant characteristics are related to network characteristics and given in table 1 .

Table 1 Correlation of ACO and VANET

\begin{tabular}{|c|c|c|}
\hline S.No & VANET & ACO \\
\hline 1 & Network & Environment \\
\hline 2 & Source Cluster & Ant Colony \\
\hline 3 & Forward and backward & Ant food foraging \\
\hline 4 & Destination cluster & Food source \\
\hline 5 & Neighbour clusters & Nearby sources \\
\hline 6 & Forage nodes & Employed ants \\
\hline
\end{tabular}


Journal of Information Technology and Digital World (2020)

Vol.02/ No. 03

Pages: $174-182$

https://www.irojournals.com/itdw/

DOI: https://doi.org/10.36548/jitdw.2020.3.005

Inverted ant colony optimization is one of the modified version of conventional ant colony optimization which converts the basic logic of attraction into repulsion based on pheromone level.in order to provide application oriented optimization, these inverted model is evolved. Pheromone and heuristic information are the essential features in inverted ant colony optimization. The relation between these features are given as

$$
P_{n j}^{i}(t)=\frac{\left(\tau_{n j}(t)^{\alpha}\right)\left(\mu_{n j}(t)^{\beta}\right)}{\sum_{i=1}^{m} P_{n l}^{i}\left(\tau_{n l}(t)^{\alpha}\right)\left(\mu_{n l}(t)^{\beta}\right)}
$$

where $\tau_{n j}(t)$ is the edge pheromone level at initial condition, $\mu_{n j}(t)$ represents the heuristic information, the amount of pheromone impression is given as $\alpha$ and $\beta$. The pheromone value is updated based on the movement of ants and it is given as

$$
\tau(t+1)=\tau(t) \frac{1}{n}
$$

The relation between the heuristic and pheromone is related to the relationship among the nodes in the network. The similarities between the users $i$ and $j$ and the trust process while transferring data depends on the network characteristics. The degree of relationship is obtained as

$$
\mu_{n j}=\frac{\mid \text { out-degree }(i) \text { nout-degree }(j) \mid}{\mid \text { out-degree }(i) \cup o u t-\operatorname{degree}(j) \mid}
$$

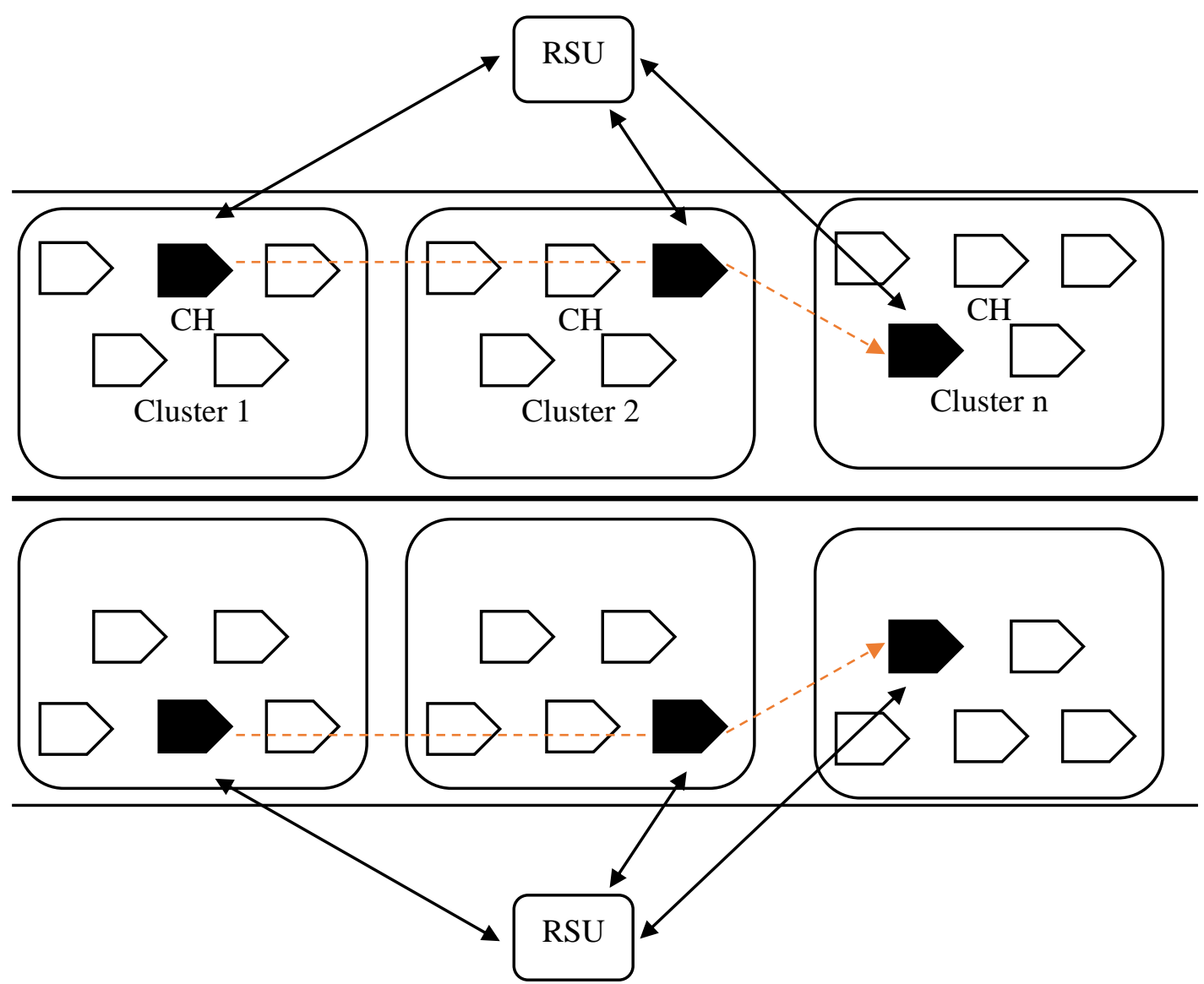

Fig. 4 Proposed Routing Model

The travel nature of ants differs in inverted ant colony optimization as the pheromone level produces aversion the second ant might not follow the first ant. The relation is mentioned based on the above probability function. If two peer nodes facilitate similar characteristics, the probability for the peer is selected randomly and the ants chooses traversed path. Figure 4 depicts the proposed model process flow which incorporates the clustering and shortest path identification using inverted ant colony optimization. 
Journal of Information Technology and Digital World (2020)

Vol.02/ No. 03

Pages: $174-182$

https://www.irojournals.com/itdw/

DOI: https://doi.org/10.36548/jitdw.2020.3.005

The steps followed in the proposed routing models is summarized as follows

1. Initially, a message is sent from the requester to its neighbour to establish a connection request. In order to obtain the connection, the peer must satisfy the necessary requirements of the nodes.

2. Once the query message is received, the neighbour node starts to respond the query. A response message is transmitted to the requester which consists of details about the node status and resource details.

3. After confirming the access through response message, requester establishes a connection with neighbour based on the probability of selection among the peer resources. The movement and selection is performed based on equation (4)

4. The movement of nodes related to ant's movement and the requirements of user are updated in neighbour table similar to pheromone update and the update is performed based on equation (5)

The above steps define the routing process in multimedia vehicle networks, and the shortest path is obtained based on inverted ant colony optimization algorithm along with clustering process.

\section{Result and Discussion}

The proposed model is experimented in eclipse environment using Java. Eclipse is an integrated development environment and a standard metrics is followed to evaluate the performance of proposed routing models. In order to validate the performance of proposed work, conventional ant colony optimization and Grey wolf optimization models are compared with the proposed model. Performance metrics are analysed for the parameters such as average waiting time, target used percentage, computation overheads, efficiency end to end delay, packet delivery ratio and throughput. The network parameters used for the simulation is listed in table 2 .

Table 2. Network simulation parameters

\begin{tabular}{|l|l|l|}
\hline S.No & Parameter & Range \\
\hline 1 & Road Length & $3 \mathrm{Km}$ \\
\hline 2 & Number of Lanes & 2 \\
\hline 3 & Number of vehicles & $25-50$ \\
\hline 4 & Transmission range & $300 \mathrm{~m}$ \\
\hline 5 & Maximum node speed & $35 \mathrm{~m} / \mathrm{s}$ \\
\hline 6 & Slot time & $10 \mu \mathrm{s}$ \\
\hline 7 & Maximum transfer power & $10 \mathrm{~mW}$ \\
\hline
\end{tabular}

The end to end delay analysis of proposed multimedia routing model is performed based on the response of resource request for data transfer, video, voice and other applications. The experimentation is performed for all the three models based on the above requests and its routing performance is analysed. Figure 5. It is observed that the performance of proposed model is much better than conventional models.

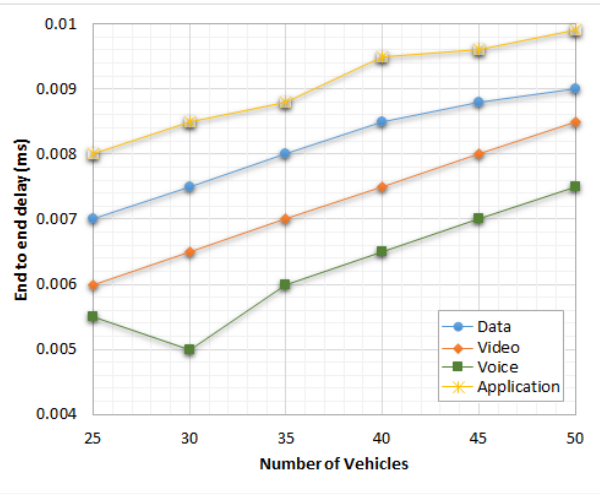

(a) Conventional ACO model

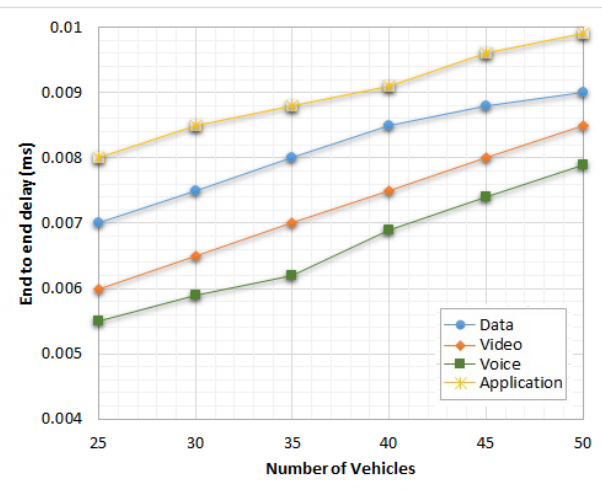

(b) Grey wolf optimization model 
Journal of Information Technology and Digital World (2020)

Vol.02/ No. 03

Pages: $174-182$

https://www.irojournals.com/itdw/

DOI: https://doi.org/10.36548/jitdw.2020.3.005

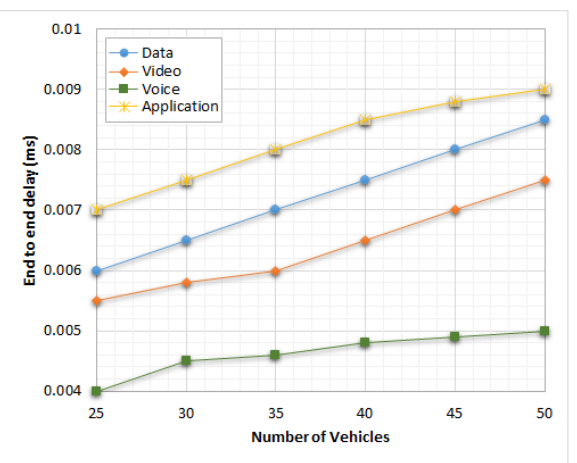

(c) Proposed k-IACO model

Fig. 5 End to end delay analysis

The throughput analysis of proposed model is computed for all the four process and an average is obtained. The average throughput is compared with the conventional models and it is observed that proposed model attains better throughput performance due to its clustering strategy.

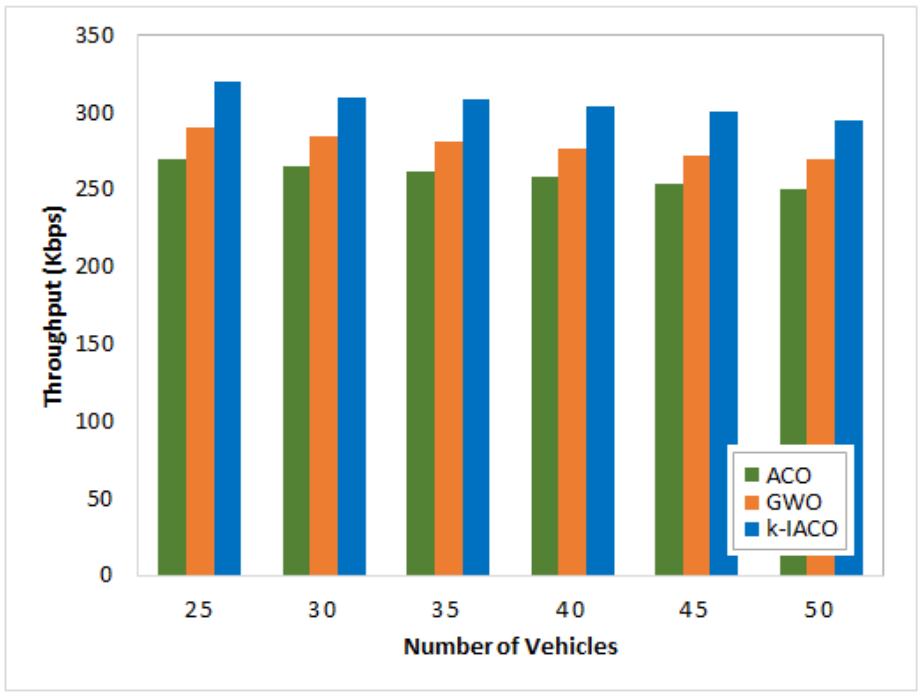

Fig.6 Average throughput comparison

Performance comparative analysis of proposed model in terms of average waiting time, target used percentage, computation overheads, efficiency are listed in table 3.

Table 3 Performance comparative analysis

\begin{tabular}{|c|c|c|c|}
\hline Parameters & ACO & GWO & k-IACO \\
\hline Average Waiting time(ms) & 10.2 & 12.4 & 5.22 \\
\hline Percentage of used targets (\%) & 86.5 & 84.2 & 96.8 \\
\hline Computation Overhead (\%) & 6.4 & 8.2 & 3.2 \\
\hline Efficiency (\%) & 94.5 & 92.6 & 97.4 \\
\hline
\end{tabular}


Journal of Information Technology and Digital World (2020)

Vol.02/ No. 03

Pages: $174-182$

https://www.irojournals.com/itdw/

DOI: https://doi.org/10.36548/jitdw.2020.3.005

It is observed from the above parameters, the performance of proposed model is much better than conventional ant colony optimization and grey wolf optimization. Proposed model attains improved efficiency over conventional ACO model due to its inverted characteristics of ant behaviour.

\section{Conclusion}

An optimal routing in multimedia vehicular networks is presented in this research work using inverted ant colony optimization and $\mathrm{k}$-means clustering. The communication in the vehicular network is framed using $\mathrm{k}$ means clustering by selecting cluster head based on its weight functions. Shortest path identification is performed based on inverted ant colony optimization. Due to continues cluster schemes the connection between the user and road side units are updated and established throughout the movement. Conventional models such as ant colony optimization and grey wolf optimization is compared with proposed model to validate the performance in terms of end to end delay, throughput, efficiency.

\section{REFERENCES}

1. Kannan, K., Devaraju, M. (2019). QoS supported adaptive and multichannel MAC protocol in vehicular adhoc network. Cluster Computing. 22:3325-3337.

2. Jiau, M. K., Huang, S. C., Hwang, J. N., \& Vasilakos, A. V. (2015). Multimedia service in cloud-based vehicular networks. IEEE Intelligent Transportation Systems Magazine, 7(3), 62-79.

3. Changqiao Xu., Quan, W., Vasilakos, A. V., Zhang, H., \& Muntean, G. M. (2017). Information-centric costefficient optimization for multimedia content delivery in mobile vehicular networks. Computer Communications, 99, 93-106.

4. Huang, C. J., Wang, Y. W., Chen, H. M., Cheng, A. L., Jian, J. J., Tsai, H. W., \& Liao, J. J. (2013). An adaptive multimedia streaming dissemination system for vehicular networks. Applied Soft Computing, 13(12), 4508-4518.

5. Kharel, J., \& Shin, S. Y. (2019). Multimedia service utilizing hierarchical fog computing for vehicular networks. Multimedia Tools and Applications, 78(7), 9405-9428.

6. Zhou Su, Yilong Hui, Qing Yang (2017). The next generation vehicular networks: A content-centric framework. IEEE Wireless Communications, 24(1), 60-66.

7. Quan, W., Song, F., Yu, C., \& Zhang, M. (2016). ICN based vehicle-to-cloud delivery for multimedia streaming in urban vehicular networks. China Communications, 13(9), 103-112.

8. Lai, C. F., Chang, Y. C., Chao, H. C., Hossain, M. S., \& Ghoneim, A. (2017). A buffer-aware QoS streaming approach for SDN-enabled 5G vehicular networks. IEEE Communications Magazine, 55(8), 68-73.

9. Yang, Q., Jiang, T., Li, W., Liu, G., Rawat, D. B., \& Wu, J. (2019). Multimedia and Social Data Processing in Vehicular Networks. Mobile Networks and Applications, 1-3.

10. Xia, Y., Chen, W., Liu, X., Zhang, L., Li, X., \& Xiang, Y. (2017). Adaptive multimedia data forwarding for privacy preservation in vehicular ad-hoc networks. IEEE Transactions on Intelligent Transportation Systems, 18(10), 2629-2641.

11. Poongodi, R. K., \& Sivakumar, T. (2018). Enhanced Adaptive Multimedia Data Forwarding for Privacy Preservation in Vehicular Ad-Hoc Networks Using Authentication Group Key. Bonfring International Journal of Software Engineering and Soft Computing, 8(1), 26-30.

12. Noura Aljeri, Azzedine Boukerche (2019). A two-tier machine learning-based handover management scheme for intelligent vehicular networks. Ad Hoc Networks, 94:1-16.

13. Salim Bitam, Abdelhamid Mellouk (2013). Bee life-based multi constraints multicast routing optimization for vehicular ad hoc networks. Journal of Network and Computer Applications, 36(3):981-991.

14. Muhammad Fahad, Farhan Aadil, Zahoor-ur- Rehman, Salabat Khan, Irfan Mehmood (2018). Grey wolf optimization based clustering algorithm for vehicular ad-hoc networks. Computers \& Electrical Engineering. 70:853-870.

15. Sadip Midya, Asmita Roy, Koushik Majumder, Santanu Phadikar (2018). Multi-objective optimization technique for resource allocation and task scheduling in vehicular cloud architecture: A hybrid adaptive nature inspired approach. Journal of Network and Computer Applications. 103:58-84.

Information Technology \&

Digital World 